Revised manuscript prepared for submission to RPD (August 10, 2016)

\title{
SPECTRAL CORRECTION FACTORS FOR CONVENTIONAL NEUTRON DOSE METERS USED IN HIGH-ENERGY NEUTRON ENVIRONMENTS - IMPROVED AND EXTENDED RESULTS BASED ON A COMPLETE SURVEY OF ALL NEUTRON SPECTRA IN IAEA-TRS-403
}

\footnotetext{
U. Oparaji ${ }^{1,2}$, Y.H. Tsai ${ }^{2}$, Y.C. Liu ${ }^{2}$, K.W. Lee ${ }^{2,3}$, E. Patelli ${ }^{1}$ and R.J. Sheu ${ }^{2,4, *}$

${ }^{1}$ Institute for Risk and Uncertainty, University of Liverpool, Liverpool L69 7ZF, United Kingdom

${ }^{2}$ Institute of Nuclear Engineering and Science, National Tsing Hua University, 101 Sec. 2, Kung Fu Road, Hsinchu, Taiwan, R.O.C.

${ }^{3}$ Institute of Nuclear Energy Research, Lungtan, Taoyuan, Taiwan, R.O.C.

${ }^{4}$ Department of Engineering and System Science, National Tsing Hua University, 101 Sec. 2, Kung Fu Road, Hsinchu, Taiwan, R.O.C.
}

* Corresponding author: Rong-Jiun Sheu (rjsheu@mx.nthu.edu.tw) 


\begin{abstract}
This paper presents improved and extended results of our previous study on corrections for conventional neutron dose meters used in environments with high-energy neutrons $\left(E_{n}>10 \mathrm{MeV}\right)$. Conventional moderated-type neutron dose meters tend to underestimate the dose contribution of high-energy neutrons because of the opposite trends of dose conversion coefficients and detection efficiencies as the neutron energy increases. A practical correction scheme was proposed based on analysis of hundreds of neutron spectra in the IAEA-TRS-403 report. By comparing ${ }^{252} \mathrm{Cf}$-calibrated dose responses with reference values derived from fluence-to-dose conversion coefficients, this study provides recommendations for neutron field characterization and the corresponding dose correction factors. Further sensitivity studies confirm the appropriateness of the proposed scheme and indicate that (1) the spectral correction factors are nearly independent of the selection of three commonly-used calibration sources: ${ }^{252} \mathrm{Cf},{ }^{241} \mathrm{Am}-\mathrm{Be}$ and ${ }^{239} \mathrm{Pu}-\mathrm{Be}$; (2) the derived correction factors for Bonner spheres of various sizes (6"-9") are similar in trend; (3) practical high-energy neutron indexes based on measurements can be established to facilitate the application of these correction factors in workplaces.
\end{abstract}

Keywords: High-energy neutrons; Neutron dose meters; Response functions; Detector calibration; Correction factors. 


\section{INTRODUCTION}

High-energy neutrons $\left(E_{n}>10 \mathrm{MeV}\right)$ are relatively penetrating and usually give a substantial dose contribution behind thick shields due to their high fluence-to-dose conversion coefficients. The accuracy of neutron dose evaluation largely depends on the knowledge of neutron energy distributions at locations of concern. However, it is generally difficult to determine the spectrum over the entire energy range from thermal up to $\mathrm{GeV}$ neutrons. Depending on the desired energy range and resolution, various neutron detectors may have to be used in combination to achieve this goal. In radiation environments with high-energy neutrons, such as at high-energy accelerator facilities, determining the relative contribution to the total dose or dose rate from high-energy neutrons and low-energy neutrons is of great interest because high-energy neutrons may have significant contribution, but only resulting in small or negligible responses in conventional type neutron monitors.

Moderated-type neutron dose meters tend to underestimate the dose contribution of high-energy neutrons because of the opposite trends of dose conversion coefficients and detection efficiencies as the neutron energy increases. The phenomenon is well known to many health physics practitioners, especially those working at high-energy accelerator facilities. Improved detector designs, such as the so-called extended-range neutron dose meters, or suggested corrections for the responses of conventional neutron dose meters have been discussed in several studies ${ }^{(1-5)}$. For example, Klett et al. ${ }^{(1)}$ showed that the response of the standard LB 6411 neutron dose meter was only $68.9 \%$ of the reference value at the CERN/CERF field with high-energy neutrons ${ }^{(6)}$. Fassò et al. ${ }^{(2)}$ demonstrated that, at high-energy electron accelerators fields, the responses of the Andersson-Braun rem meter calibrated with ${ }^{252} \mathrm{Cf}$ or $\mathrm{Am}-\mathrm{Be}$ neutron 
sources underestimated ambient dose equivalent by approximately $30 \%$ to $60 \%$ for concrete of thicknesses varying from 60 to $120 \mathrm{~cm}$. Regarding the development of extended-range neutron dose meters, Olsher et al. ${ }^{(3)}$ introduced an improved neutron rem meter called WENDI and Berthold Technologies ${ }^{(1)}$ presented a new version of LB6411 neutron dose monitor. Both detectors are capable of detecting neutrons with energies up to $\mathrm{GeV}$ by the use of lead or tungsten in moderator as a neutron multiplier. These extended-range neutron dose meters are relatively expensive and considerably heavy compared with the original designs due to the embedded heavy metal inside the detectors.

To have proper dose estimation in high-energy neutron environments, in addition to using the extended-range neutron dose meters, it is possible and of interest to correct the underestimated responses of conventional detectors. As an example, according to the characteristics of neutron spectra behind thick shields at high-energy electron accelerators ${ }^{(2)}$, a practical approach adopted at SLAC was simply doubling the rem meter readings in neutron measurements. Jagerhofer et al. ${ }^{(4)}$ demonstrated that the WEND-II rem meter is an appropriate device to monitor ambient dose equivalent rates in high-energy neutron dominated fields. In addition, Monte Carlo simulations were used to determine field specific correction factors for the ${ }^{252} \mathrm{Cf}$-calibrated WEND-II, which resulted in a better agreement between calculations and measurements. In our previous study ${ }^{(5)}$, the effect of the neutron spectrum on the accuracy of dose measurements was systematically investigated by considering a set of ten selected neutron spectra representing various neutron environments. A simple correction scheme was provided for users to correct the dose underestimation of conventional neutron dose meters used in radiation fields with high-energy neutrons. The magnitude of correction is spectrum-dependent and described as a function of the 
estimated flux percentage of high-energy neutrons in the spectrum of workplace or a spectral index based on in-situ measurements of two designated Bonner spheres. However, neutron spectra typically span several orders of magnitude and vary widely from place to place. A serious concern about the validity of the correction scheme mainly originated from the ten selected neutron spectra, where the correction factors and fitting formulas were derived. In addition, the neutron detector and calibration source adopted in the previous study might have certain effect on the estimation of dose responses and the corresponding correction factors. Can the derived correction scheme be universally extended to environments having completely different neutron spectra? To address these issues and preclude the subjective selection of neutron spectra for analysis, this study presented improved and extended results based on a complete survey of over 200 neutron spectra collected in the IAEA Technical Reports Series No. 403 (IAEA-TRS-403) (7) $^{(1)}$ a series of sensitivity studies.

\section{MATERIALS AND METHODS}

\section{Bonner spheres and neutron dose meters}

Conventional neutron dose meters such as popular 9-inch rem balls and Andersson-Braun rem meters are widely used for neutron surveillance or area monitoring in workplaces. These moderated-type devices present a reasonable fit between the detection efficiency and the fluence-to-dose conversion coefficients over a wide range of neutron energies. It has been well known that the detectors based solely on moderating or absorbing materials to shape the response function suffer from no effective response to high-energy neutrons. By embedding heavy metals in neutron moderators, the effective detector response can be extended to the $\mathrm{GeV}$ range, such as the two extended-range neutron rem meters $\mathrm{WENDI}^{(3)}$ and $\mathrm{LB} 6411^{(1)}$ that 
mentioned previously. However, these commercial neutron dose meters, either conventional or extended-range types, were not selected for being the targets of this study because we did not have enough details in their designs including dimensions and material compositions. These details are necessary for detector modelling in numerical simulations to have an accurate prediction of the detector response function, one of the key ingredients in this study.

The Bonner sphere spectrometer is widely used in neutron spectrum determination because of several advantages including a wide energy range, isotropic angular response, reasonable detection sensitivity and excellent neutron-gamma discrimination. Intermediate-sized Bonner spheres are in principle similar to the design of most conventional neutron dose meters. More importantly, the specification of the spectrometer provides detailed information for high-fidelity response function calculations. The PTB neutron multi-sphere spectrometer (NEMUS) ${ }^{(8)}$ was used in this study, consisting of 11 standard polyethylene spheres of various diameters and four extended-range spheres. The four extended-range spheres were labeled 4C5_7, 3P5_7, 4P5_7, and 4P6_8; the three numbers in the label indicate, respectively, the diameters of the three spherical layers in inches: the inner polyethylene sphere, the embedded copper (C) or lead (P) shell, and the outer polyethylene sphere. Among the Bonner spheres of various sizes and designs, two spheres were selected (the standard 9" sphere and the 4P6_8 lead-embedded sphere) representing the conventional and extended-range neutron dose meters, respectively. In addition, standard Bonner spheres of diameters of 6", 7" and 8" were considered as alternative candidates of conventional type neutron dose meters in order to investigate the effect of various choices of neutron dose meters on the resulting correction factors. The response functions of Bonner spheres were calculated using the continuous-energy Monte 
Carlo transport code $\mathrm{MCNPX}^{(9)}$. For more details on the response function calculations and validation, please refer to our previous papers ${ }^{(5,10)}$. Figure 1 shows the calculated response functions of five standard Bonner spheres of diameters ranging from 5" to 9" and two extended-range spheres 3P5_7 and 4P6_8. These detectors were used in this study as possible candidates of neutron dose meters or as instruments to construct a spectral indicator of neutron field.

\section{Neutron spectra and dose correction factors}

Reliable neutron dose measurement is difficult because of the wide range of neutrons and the imperfect response of most detectors. Thus, an instrument calibration is important, which requires a calibration field of similar characteristics and appropriate calibration procedure ${ }^{(11)}$. In practice, neutron dose meters used for radiation protection purpose are commonly calibrated with ${ }^{252} \mathrm{Cf}$ or other standard sources and then used in various workplaces. However, because of the limited energy range of a calibration source, calibrated dose meters are actually not recommended for use in neutron fields exhibiting characteristics that differ substantially from the calibration source. If so, one should be cautious in the detector response and a workplace-specific or spectrum-dependent correction factor may be necessary, especially for the problem of dose underestimation caused by high-energy neutrons.

The approach adopted in this study for the estimation of spectral correction factors focused on three aspects of neutron dose measurement: detector calibration, response function and dose evaluation. First, the Bonner sphere chosen as the dose meter was irradiated in a well-defined neutron field produced by a traceable standard

source ${ }^{252} \mathrm{Cf}$. The dose calibration factor (DCF) of the detector expressed in unit of $\mu \mathrm{Sv} / \mathrm{h} / \mathrm{cps}$ can be determined by dividing the known dose rate at the location by the 
recorded net counting rate. The term dose or dose rate in this paper refers to the operational quantity of the ambient dose equivalent, $H^{*}(10)$. Second, by folding the neutron spectrum under consideration with the detector response function, the neutron counting rate of the detector can be estimated and further converted into the neutron dose rate. This dose rate was denoted as $\mathrm{H}^{*}(10)_{\mathrm{cf} 252}$ because the conversion was based on the detector calibration using a ${ }^{252} \mathrm{Cf}$ neutron source. Third, the neutron dose rate at the location of interest can be evaluated by a parallel and more rigorous process, which is a direct folding of the fluence-to-dose conversion factors with the spectrum. This dose rate denoted as $\mathrm{H}^{*}(10)_{\text {spe }}$ directly corresponds to the neutron spectrum under consideration. The ICRP-74 ${ }^{(12)}$ conversion coefficients for the ambient dose equivalent were adopted for neutron energies below $180 \mathrm{MeV}$, and the high-energy extensions calculated by Pelliccioni ${ }^{(13)}$ were concatenated to cover neutrons of higher energies. A comparison of the dose rates derived from the two processes leads to a spectrum-dependent correction factor for the neutron dose meter, defined as the ratio of $\mathrm{H}^{*}(10)_{\mathrm{spe}}$ to $\mathrm{H}^{*}(10)_{\mathrm{c} 252}$. By this definition, the correction factors for neutron spectra similar to that of ${ }^{252} \mathrm{Cf}$ must be close to 1.0 .

For an ideal neutron dose meter, the correction factor for any given spectrum always approaches to 1.0. No spectrum-dependent correction is needed because of a perfect match between the detector response function and fluence-to-dose conversion coefficients over the entire neutron energy range. The condition obviously does not hold in reality, and in particular for high-energy neutrons. Therefore, any deviation of the calculated correction factor from the ideal value of 1.0 indicates certain spectral effect on the response of a ${ }^{252} \mathrm{Cf}$-calibrated neutron dose meter. Through a systematic study of this effect, the relationship between the neutron field characterization and the dose response of a ${ }^{252} \mathrm{Cf}$-calibrated detector can be derived accordingly. Compared 
with our previous result ${ }^{(5)}$, the value of this work lies in providing an in-depth analysis of spectral correction factors based on a much larger database and sensitivity studies of key parameters. The result leads to a more rigorous and useful correction scheme than that previously provided. This study examined the spectral effect through a complete survey of all neutron spectra in the IAEA-TRS-403 report ${ }^{(7)}$, rather than limited to the ten neutron spectra that were selected subjectively.

The IAEA report contains a large number of neutron spectra collected from various literature sources, including neutron spectra in natural environments, neutron spectra used for instrument calibration, and neutron spectra that are representative of fields in various facilities involving neutron sources or neutron-generating devices, such as nuclear power plants, medical accelerators, and high-energy accelerators. Among the total 243 neutron spectra being investigated, a total of 146 spectra in the database contains certain portions of high-energy neutrons $\left(E_{n}>10 \mathrm{MeV}\right)$, ranging from a small flux percentage to $70 \%$. Among these spectra with high-energy neutrons, 31 of them are of the most interest in the analysis because an appreciable portion of high-energy neutrons, say $\geq 10 \%$, is involved in radiation fields. In addition to that, thousands of new spectra were generated by a random linear combination of those spectra in the database in order to test and verify the suggested correction scheme. The correction, in essence, largely depends on the characteristics of neutron energy distribution. A systematic analysis of all these neutron spectra was performed on the basis of the detector response function and neutron field characterization.

\section{RESULTS AND DISCUSSION}




\section{Neutron field characterization}

The field characterization attempted to reasonably account for the contribution of high-energy neutrons that cannot be properly reflected by conventional neutron dose meters. If the spectrum at the location of interest is known, it is straightforward to characterize the field in terms of the flux percentage of neutrons with energies greater than $10 \mathrm{MeV}$. However, if the spectrum is unknown, which is usually the case in most situations, performing radiation transport calculations or in-situ measurements are inevitable to be able to grasp some information about neutron energy distribution at the location. Alternative spectral indices that replaces the flux percentage of high-energy neutrons have to be established. In our previous study ${ }^{(5)}$, the pair of an extended-range sphere 4P6_8 and a standard 6" sphere was selected for the purpose of constructing a spectral index, indicating the significance of high-energy neutrons in workplaces. The selection was based on an observation that the response functions of the 4P6_8 and 6" spheres are nearly overlap for low-energy neutrons and deviate substantially for neutron energies above $10 \mathrm{MeV}$ (see Fig.1). After exploring the response functions of all Bonner sphere configurations, another pair of Bonner spheres were identified, the extended-range 3P5_7 and standard 5", which shows similar characteristics in their response functions (Fig. 1). Therefore, the ratio between the measured counting rates of this pair of spheres could also be served as a reasonable indicator of high-energy neutrons in radiation field.

Among available configurations in the NEMUS spectrometer, further study summarized three requirements for being a good pair of Bonner spheres that can be used to construct a practical index for high-energy neutron characterization. The first requirement is straightforward that the extended-range sphere must have the same 
thickness of polyethylene as that of the standard-type sphere, ensuring a similar response for neutron energies below $10 \mathrm{MeV}$. Second, the extended-range spheres should have lead embedded in the moderator rather than copper because of its insufficiency in neutron multiplicity. This can be observed by comparing the response functions of lead- and copper-embedded Bonner spheres, confirming that copper is not a good material for neutron multiplication to properly reflect the dose contribution of high-energy neutrons. The third requirement is about the thickness of the embedded lead in neutron moderator. It cannot be too thin, according to our study, one-inch-thick lead is suggested in order to have sufficient response to high-energy neutrons. The combinations 4P6_8/6" and 3P5_7/5" were found available and suitable for this purpose, while other combinations fail these requirements and are not suggested.

\section{General trends of spectral correction factors}

Following the dose comparison procedure described previously, a correction factor can be obtained for each neutron spectrum in the environment where the ${ }^{252} \mathrm{Cf}$-calibrated neutron dose meter is to be used. This spectrum-dependent correction factor, denoted as $\mathrm{H}^{*}(10)_{\mathrm{spe}} / \mathrm{H}^{*}(10)_{\mathrm{cf} 252}$, is the ratio of the ambient dose equivalent rate calculated by folding the spectrum directly with the fluence-to-dose conversion coefficients to that delivered by the detector calibrated with ${ }^{252} \mathrm{Cf}$. Considering the standard 9" sphere as a neutron dose meter, Fig. 2 shows the distribution of dose correction factors as a function of the flux percentage of high-energy neutrons in the spectrum. Each data point in the figure represents a specific neutron spectrum collected from the IAEA-TRS-403 report. For those spectra without high-energy neutrons, the dose correction factors are all less than 1.0 to varying extents. This reflects a well-known phenomenon that the response functions of conventional 
neutron dose meters tend to overestimate the magnitude of fluence-to-dose conversion coefficients for neutrons in intermediate energy range. A conservative estimate of the neutron dose in workplace is acceptable for radiation protection purposes. However, as shown in Fig. 2, the dose correction factors for the standard 9" sphere used in radiation fields with high-energy neutrons may range from 1.0 up to more than 3.0, indicating significant dose underestimation that cannot be ignored. On the other hand, a repeated analysis was performed by replacing the standard 9" sphere with the extended-range 4P6_8 sphere. The result is shown in Fig. 3. As expected, most of the dose correction factors are close to or less than 1.0, indicating a satisfactory performance or at least conservative responses of this extended-range dose meter when exposed in various radiation fields with high-energy neutrons.

The dose correction factors in Fig. 2 in general show a monotonically increasing trend as a function of the flux percentage of high-energy neutrons, which enables us to propose a practical correction scheme for conventional neutron dose meters used in high-energy neutron environments. Base on the method of least squares, a curve fitting by a second order polynomial was performed to establish the relationship between the dose correction factor and the high-energy neutron percentage in a spectrum. Only those spectra in IAEA-TRS-403 with appreciable component of high-energy neutrons, say $\geq 10 \%$ in flux percentage, were considered in the curve fitting process. The equation of the curve was forced to pass through the given point $(0,1)$ in order to meet the purpose of the correction factor in the context of phenomena discussed in this paper. The resulting equation obtained is shown in Fig. 2 and compared with our previous work ${ }^{(5)}$, which was obtained based on an analysis of ten selected neutron spectra representing various workplaces of interest. The difference of the two fitting curves is relatively small when compared with the overall magnitude of 
correction. For example, the difference between two derived correction factors is only about $2 \%$ for a case of neutron field having $50 \%$ high-energy neutrons, which overall corresponds to a factor of 2 correction in neutron dose estimation. Nevertheless, the new fitting curve is suggested for practical use because it was derived from an enlarged collection of neutron spectra at various workplaces.

Before applying the correction scheme in Fig. 2 to determine a dose correction factor for the 9"-sphere responses, it is necessary to have an estimate of the flux percentage of high-energy neutrons in the neutron field. This is impractical without the information of neutron energy distribution at the location. Neutron spectrum determination in workplaces is a difficult task, time-consuming and needs expertise. In our previous study ${ }^{(5)}$, we proposed a practical approach as an alternative to estimate the dose correction factor based on the ratio of the measured responses of two Bonner spheres (4P6_8 sphere vs. 6"). Comparing the characteristics of their response functions, this ratio can provide an indication of the significance of high-energy neutrons in a neutron field. Figure 4, which is similar to Fig. 2, presents the dose correction factors for the 9" sphere when used as a neutron dose meter in various neutron environments. The spectrum index in the abscissa, rather than the flux percentage of high-energy neutrons, has been replaced by the ratio between measured responses of the 4P6_8 and 6" spheres. The larger the ratio between the two detectors' responses, the more high-energy neutrons at the location. As expected, the dose correction factors exhibit a gradually increasing trend as a function of the new spectral index. A linear curve fitting was suggested by observing the distribution of these correction factors. If necessary, the resulting equation in Fig. 4 can provide guidance to health physicists on the proper correction of the responses of conventional neutron dose meters. Note that a linear fitting equation was used in this case instead of a 
second-order polynomial that we proposed in our previous study ${ }^{(5)}$. As compared in Fig. 4, the difference between the two fitting curves is within $\pm 10 \%$ in the whole range of the spectral indexes from 1.0 to 3.1 , representing all the neutron spectra in the IAEA-TRS-403 report.

\section{Neutron calibration sources and spectral correction factors}

The proposed correction scheme in Figs. 2 and 4 was obtained assuming that the dose meters were calibrated by a ${ }^{252} \mathrm{Cf}$ neutron source. The energy spectrum of spontaneous fission neutrons from a ${ }^{252} \mathrm{Cf}$ source can be characterized by a Maxwellian distribution and peaks at about $2 \mathrm{MeV} .{ }^{241} \mathrm{Am}-\mathrm{Be}$ and ${ }^{239} \mathrm{Pu}-\mathrm{Be}$ are also commonly used neutron sources in detector calibration. Note that the two $\operatorname{Be}(\alpha, n)$ sources exhibit complicated spectra with multiple peaks at about $3.5,5$, and $8 \mathrm{MeV}$ and have higher average energies of approximately 3-4 MeV. An important question arose as to what would happen to the suggested correction factors if one used different neutron sources to calibrate the dose meters.

To answer this question, the procedure previously described to determine the spectral correction factors was additionally repeated twice but using ${ }^{241} \mathrm{Am}-\mathrm{Be}$ and

${ }^{239} \mathrm{Pu}-\mathrm{Be}$, respectively, in place of the original calibration source ${ }^{252} \mathrm{Cf}$. Considering the same 9" sphere as a neutron dose meter, Fig. 5 shows a comparison of three fitting curves of dose correction factors corresponding to three different calibration sources $\left({ }^{252} \mathrm{Cf},{ }^{241} \mathrm{Am}-\mathrm{Be}\right.$, and $\left.{ }^{239} \mathrm{Pu}-\mathrm{Be}\right)$. These curves represent the suggested dose correction factors as a function of the flux percentage of high-energy neutrons in the spectrum. The data points in Fig. 5 are spectral correction factors calculated for a ${ }^{252}$ Cf-calibrated detector (same as those in Fig. 2). The other two sets of spectral 
correction factors calculated for the detector calibrated by ${ }^{241} \mathrm{Am}-\mathrm{Be}$ and ${ }^{239} \mathrm{Pu}-\mathrm{Be}$, respectively, are similar and omitted here, only the resulting curves are presented in the figure for clear comparison. The result in Fig. 5 indicates that the dose correction factors are mainly a property of neutron field and not sensitive to the selection of these three commonly-used calibration sources, which is a favorable outcome in practical application of these spectral correction factors.

\section{Neutron dose meters and spectral correction factors}

The spectral correction factors in Figs. 2 and 4 were generated using the 9" Bonner sphere as a neutron dose meter. However, there are many moderated-type neutron dose meters commercially available and widely used in numerous facilities. What if one uses another dose meter with a somewhat different response function from that of the 9" sphere? Is the proposed correction scheme still suitable in practice? To partly address this issue, the previous procedure used to determine the spectral correction factors was repeated for neutron dose meters showing different response functions. In addition to the popular 9" sphere, three medium-sized Bonner spheres (6", 7" and 8") were purposely selected to represent neutron dose meters of similar type but with different response functions (Fig. 1). The resulting dose correction factors were analyzed and compared. As a function of the defined spectral index of high-energy neutrons in workplaces, Fig. 6 gives a comparison of four fitting curves corresponding to four neutron dose meters (6", 7", 8" and 9") under consideration. Again, the data points in the figure are spectral correction factors of the 9" sphere, the rest of the data points are omitted for clarity.

The four fitting curves in Fig. 6 are similar in trend with slopes varying from 
0.816 to 1.010 . Except for the 6" sphere, the spectral correction curves of the 7", 8 ", and 9" spheres are almost consistent with each other. Comparing with the overall magnitude of the dose correction, one can conclude that the differences in these correction curves are relatively minor. For example, the difference between the resulting correction factors of two extreme spheres (6" vs. 9") is only about $10 \%$ even for a neutron field with a high spectral index of 3.0, indicating a significant flux percentage, approximately $65 \%$, of high-energy neutrons. This observation to some extent confirmed the dose correction scheme proposed for accounting for the contribution of high-energy neutrons is dominantly a property of neutron field under consideration and only shows minor dependencies on the calibration sources and dose meters used in practical measurements. This is why sometimes we call it the "spectral correction factor" in this study.

\section{Verification of the proposed correction scheme}

Although the IAEA-TRS-403 report contains a large collection of neutron spectra available in literature, it still does not exhaust the possibility of neutron spectra in workplaces. To verify the universal validity of the proposed correction scheme, artificial spectra were generated by an algorithm and then the corresponding correction factors derived. Artificial spectra are created by randomly selecting two neutron spectra $\phi_{i}(E)$ and $\phi_{j}(E)$ with appreciable flux percentage $(\geq 10 \%)$ of high-energy neutrons from the IAEA-TRS-403 collection. The two spectra are normalized and then superimposed by applying a randomly-generated weighting factor $w$ and its additive inverse $(1-w)$, respectively. By this way, all the generated new spectra could be considered at least physically meaningful and are suitable for testing the appropriateness of the proposed correction scheme. 
Figures 7 and 8 verify the validity of the proposed curve fitting in Figs. 2 and 4, respectively. The verification process of both curves was carried out by generating randomly 1000 neutron spectra representing various workplaces. Each spectrum can derive a spectral correction factor used to correct the underestimated dose response of a ${ }^{252}$ Cf-calibrated 9" Bonner sphere to high-energy neutrons. From the results shown in Figs. 7 and 8, there is a consistent trend between the predicted curve and the spectral correction factors corresponding to those randomly generated neutron spectra. The margin of error of the proposed fitting curve in Fig. 7 is within $\pm 10 \%$ as $90 \%$ of the randomly generated spectral correction factors fall within the error range, while the margin of error of the proposed fitting curve in Fig. 8 is slightly larger but still within $\pm 15 \%$. Hence, we can conclude that the proposed correction scheme matches specifications and assumptions considered acceptable for the given purpose of application.

\section{CONCLUSION}

For radiation protection purpose, moderated-type neutron dose meters are routinely calibrated with standard neutron sources such as ${ }^{252} \mathrm{Cf}$ and used in various locations or environments. A well-known issue regarding conventional designs based solely on neutron moderation is the dose underestimation in the presence of high-energy neutrons, resulting from the intrinsic inconsistency between the low detection efficiency and high dose contribution of high-energy neutrons. This paper presents a practical scheme for correcting the dose underestimation of conventional neutron detectors in radiation environments with high-energy neutrons and additionally confirms a satisfactory performance of extended-range neutron dose 
meters in similar conditions. The necessary correction could be significant, ranging from 1 (no correction) to more than a factor of 3 depending on the extent to which high-energy neutrons are present in radiation fields. The correction requires first a proper neutron field characterization, either in terms of the flux percentage or some spectral indexes based on in-situ measurements, accounting for the significance of high-energy neutrons at the location. Fitting curves of the dose correction factors for a ${ }^{252}$ Cf-calibrated 9"-sphere dose meter were reported, as a function of the flux percentage of high-energy neutrons in the spectrum and as a function of the ratio between the measured responses of two Bonner spheres (4P6_8 versus 6").

In addition to improved fitting results based on a large collection of neutron spectra, this study addressed two important questions associated with the applications of this correction scheme in practical situations where different calibration sources or dose meters are used. The sensitivity study found that different choices among three commonly-used calibration sources ${ }^{252} \mathrm{Cf},{ }^{241} \mathrm{Am}-\mathrm{Be}$, and ${ }^{239} \mathrm{Pu}-\mathrm{Be}$ only have little effect on the values of the correction factors and the correction factors for Bonner spheres of different sizes (6", 7", 8" and 9") do not change substantially, which implies the correction factor tends to be a property of the neutron field rather than a property that strongly depends on the details of a moderated-type neutron dose meter. These observations practically facilitate the implementation and application of the suggested spectrum-dependent dose correction factors in workplaces. 


\section{ACKNOWLEDGEMENTS}

The authors thank the funding support of Ministry of Science and Technology in

Taiwan (NSC 102-2221-E-007-091-MY3) and Institute of Nuclear Energy Research (NL1040697). 


\section{REFERENCES}

1. Klett, A., Mayer, S., Theis, C., Vincke, H. A neutron dose rate monitor for high energies. Rad. Meas. 41, S279-S282 (2007).

2. Fassò, A., Liu, J.C., Rokni, S.H. Neutron spectra and dosimetric quantities outside typical concrete shielding of synchrotron facilities. ICRS-12 \& RPSD-2012, Nara, Japan, 2-7 September 2012, paper ID 2C-21.

3. Olsher, R.H., Hsu, H.H., Beverding, A., Kleck, J.H., Casson, W.H., Vasilik, D.G., Devine, R.T. WENDI: an improved neutron rem meter. Health Phys. 79, 170-181 (2000).

4. Jagerhofer, L., Feldbaumer, E., Forkel-Wirth, D., Theis, C., Vincke, H., Iwamoto, Y., Hagiwara, M., Satoh, D., Iwase, H., Yashima, H. Matsumoto, T., Masuda, A., Nishiyama, J., Nakamura, T., Sato, T., Nakane, Y., Nakashima, H., Sakamoto, Y., Tamii, A., Hatanaka, K. Characterization of the WENDI-II REM counter for its application at MedAustron. Prog. Nucl. Sci. Technol. 2, 258-262 (2011).

5. Lee, K.W., Sheu, R.J. Spectral correction factors for conventional neutron dose meters used in high-energy neutron environments. Radiat. Prot. Dosim. 164, 210-218 (2015).

6. Mitaroff, A., Silari, M. The CERN-EU high-energy reference field (CERF) facility for dosimetry at commercial flight altitudes and in space. Radiat. Prot. Dosim. 102, 7-22 (2002).

7. International Atomic Energy Agency. Compendium of neutron spectra and detector responses for radiation protection purposes. Technical Reports Series No. 403 (Vienna: IAEA) (2001).

8. Wiegel, B., Alevera, A.V. NEMUS-the PTB neutron multisphere spectrometer: Bonner spheres and more. Nucl. Instrum. Meth. A 476, 36-42 (2002).

9. Pelowitz, D.B. (Ed.) MCNPX user's manual, Version 2.7.0, LA-CP-11-00438, 
Los Alamos National Laboratory (Los Alamos, NM, USA) (2011).

10. Lee, K.W., Yuan, M.C., Jiang, S.H., Sheu, R.J. Comparing standard Bonner spheres and high-sensitivity Bonner Cylinders. Radiat. Prot. Dosim. 161, 233-236 (2014).

11. Schuhmacher, A. Neutron calibration facilities. Radiat. Prot. Dosim. 110, 33-42 (2004).

12. International Commission on Radiological Protection. Conversion coefficients for use in radiological protection against external radiation. ICRP Publication 74 (Oxford, UK: Pergamon Press) (1996).

13. Pelliccioni, M. Overview of fluence-to-effective dose and fluence-to-ambient dose equivalent conversion coefficients for high energy radiation calculated using the FLUKA code. Radiat. Prot. Dosim. 88, 279-297 (2000). 


\section{Figures' numbers and their captions}

Figure 1.

Response functions of five standard Bonner spheres (5" to 9") and two extended-range spheres (3P5_7 and 4P6_8).

Figure 2 .

Approximation of the spectrum-dependent dose correction factors for the 9" Bonner sphere (calibrated with ${ }^{252} \mathrm{Cf}$ ) using the flux percentage of high-energy neutrons in the spectrum and a comparison with our previous result (RPDv164p210).

Figure 3.

Spectrum-dependent dose correction factors for the 4P6_8 Bonner sphere (calibrated with ${ }^{252} \mathrm{Cf}$ ) as a function of the flux percentage of high-energy neutrons in the spectrum.

Figure 4.

Approximation of the spectrum-dependent dose correction factors for the 9" Bonner sphere (calibrated with ${ }^{252} \mathrm{Cf}$ ) using the ratio between the responses of two Bonner spheres (4P6_8 versus 6") and a comparison with our previous result (RPDv164p210).

Figure 5 .

Comparison of the spectrum-dependent dose correction factors for the 9" Bonner sphere calibrated with ${ }^{252} \mathrm{Cf},{ }^{241} \mathrm{Am}-\mathrm{Be}$, and ${ }^{239} \mathrm{Pu}-\mathrm{Am}$ neutron sources.

Figure 6.

Comparison of the spectrum-dependent dose correction factors for four Bonner spheres (6", 7", 8", and 9") calibrated with a ${ }^{252} \mathrm{Cf}$ neutron source.

Figure 7.

Verification of the proposed curve fitting in Fig. 2 by considering 1000 randomly generated neutron spectra representing various workplaces.

Figure 8.

Verification of the proposed curve fitting in Fig. 4 by considering 1000 randomly generated neutron spectra representing various workplaces. 
Figure 1.

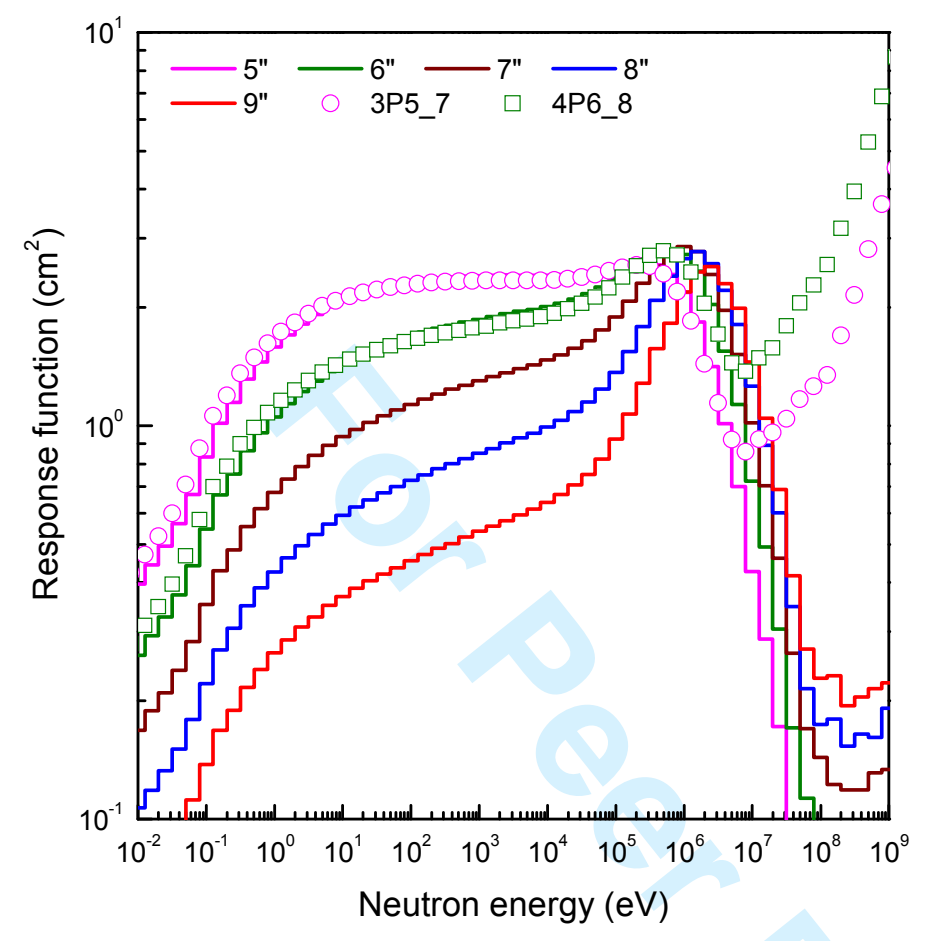


Figure 2.

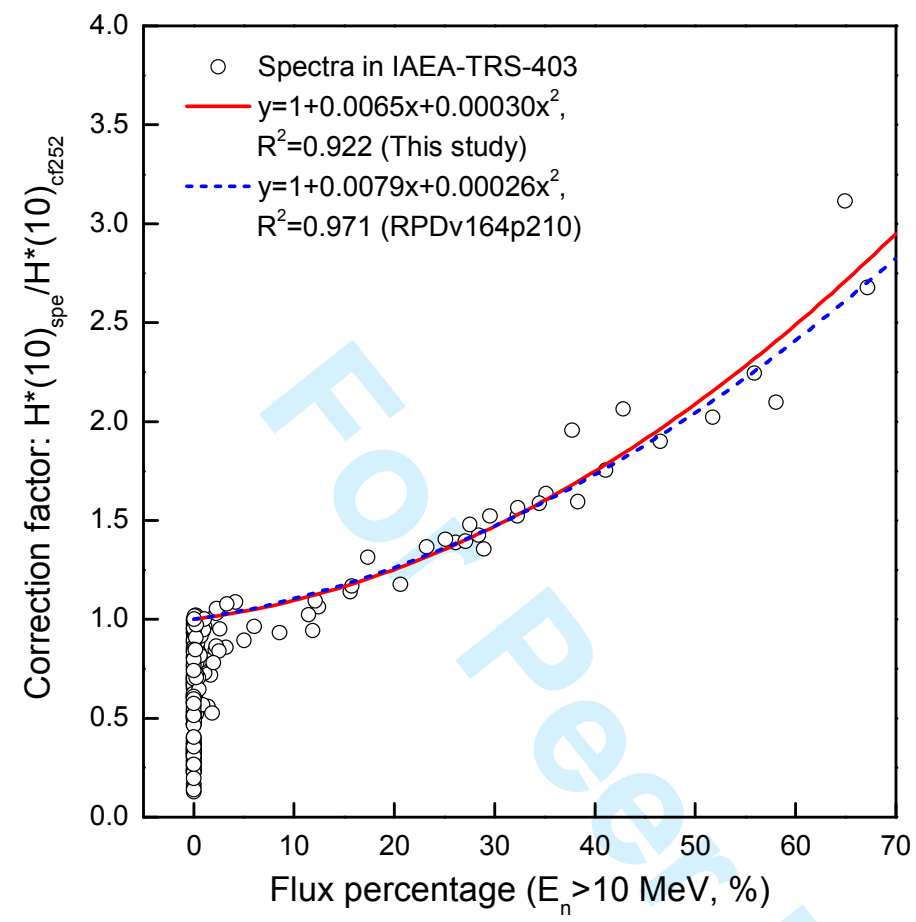


Figure 3.

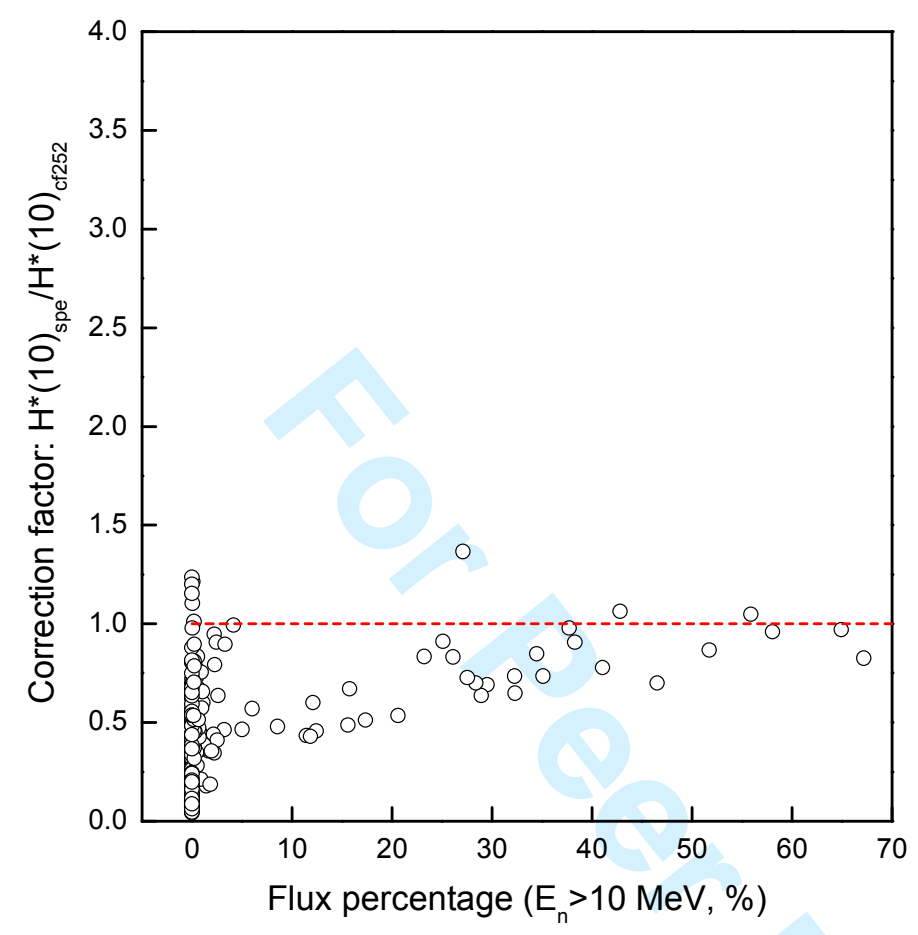


Figure 4.

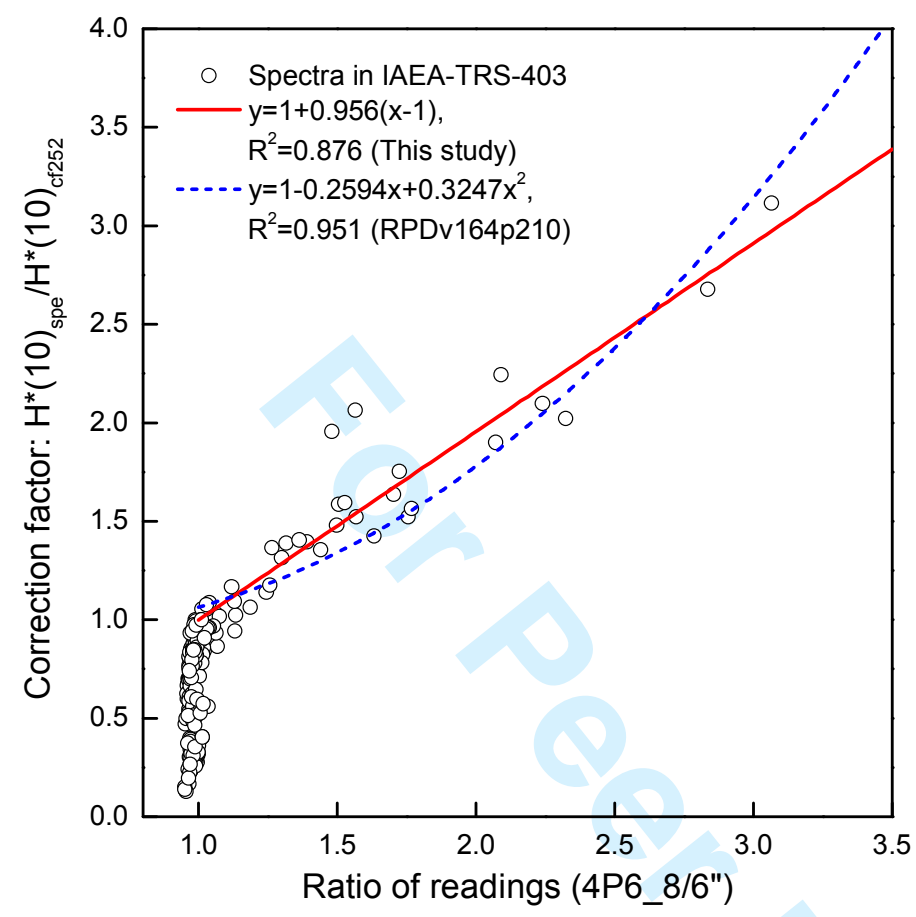


Figure 5.

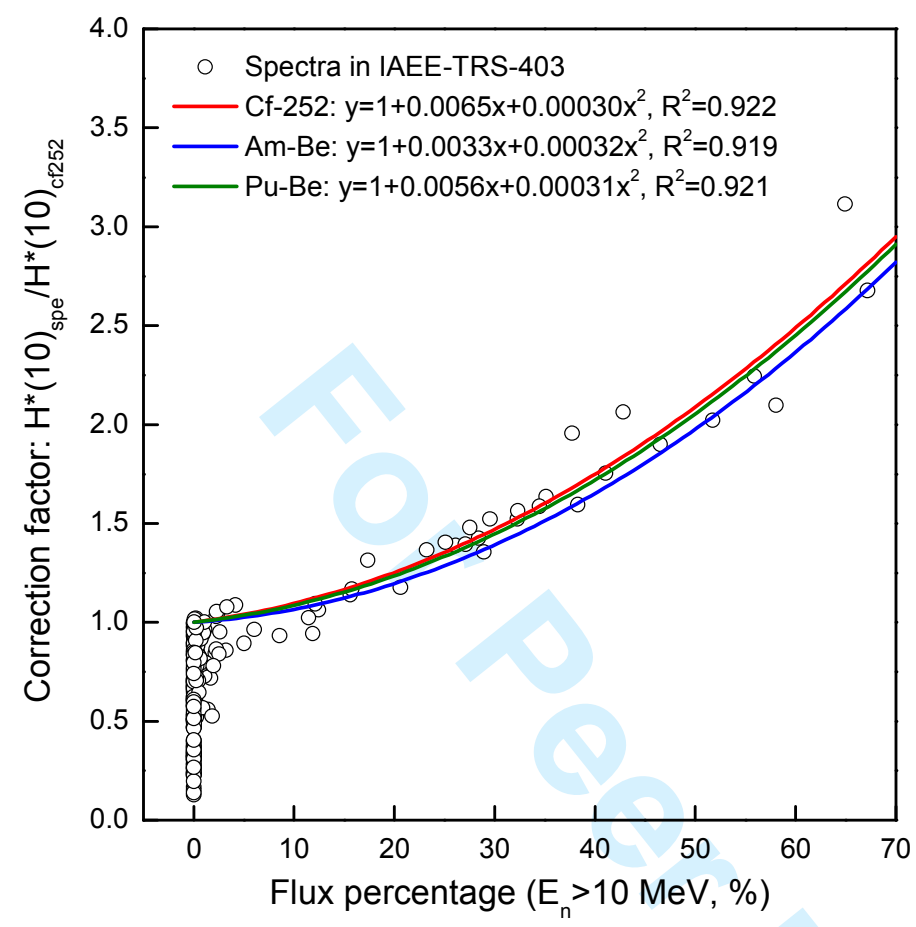


Figure 6.

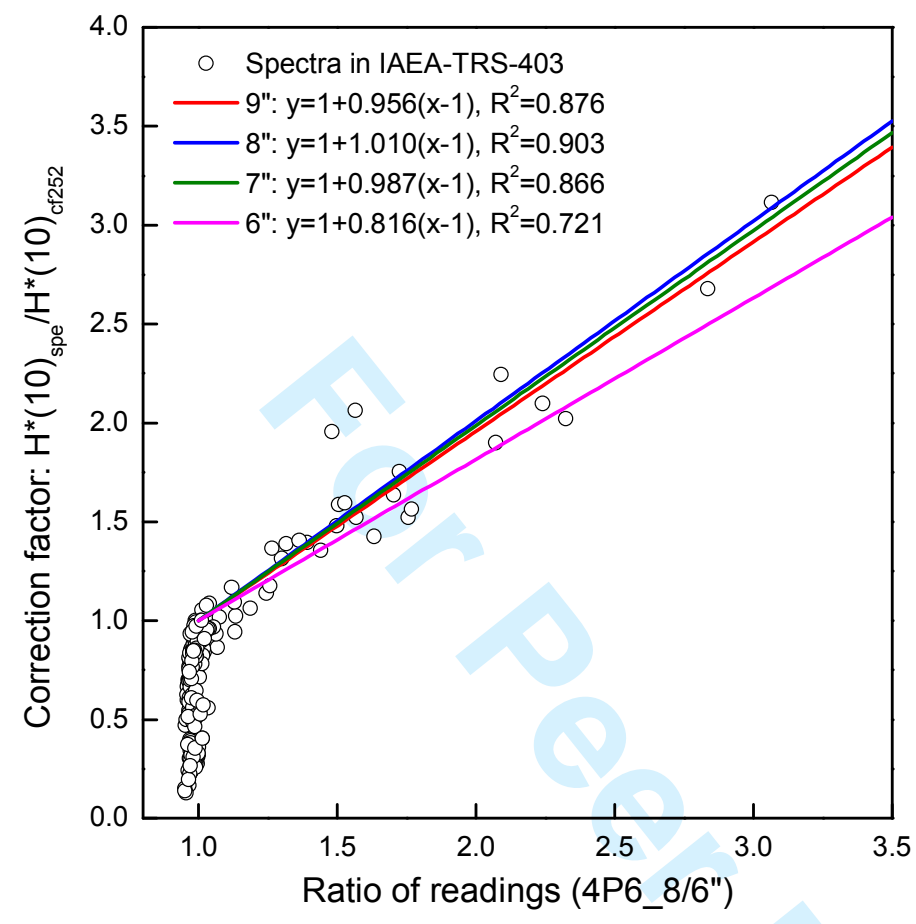


Figure 7.

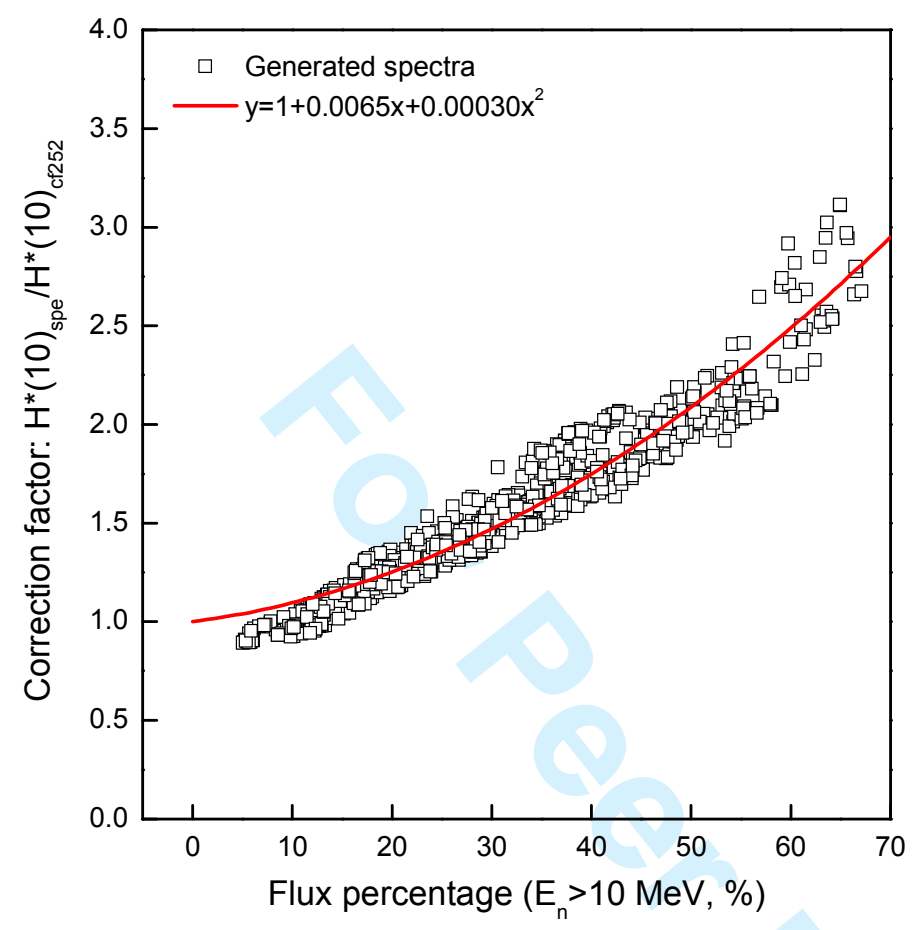


Figure 8.

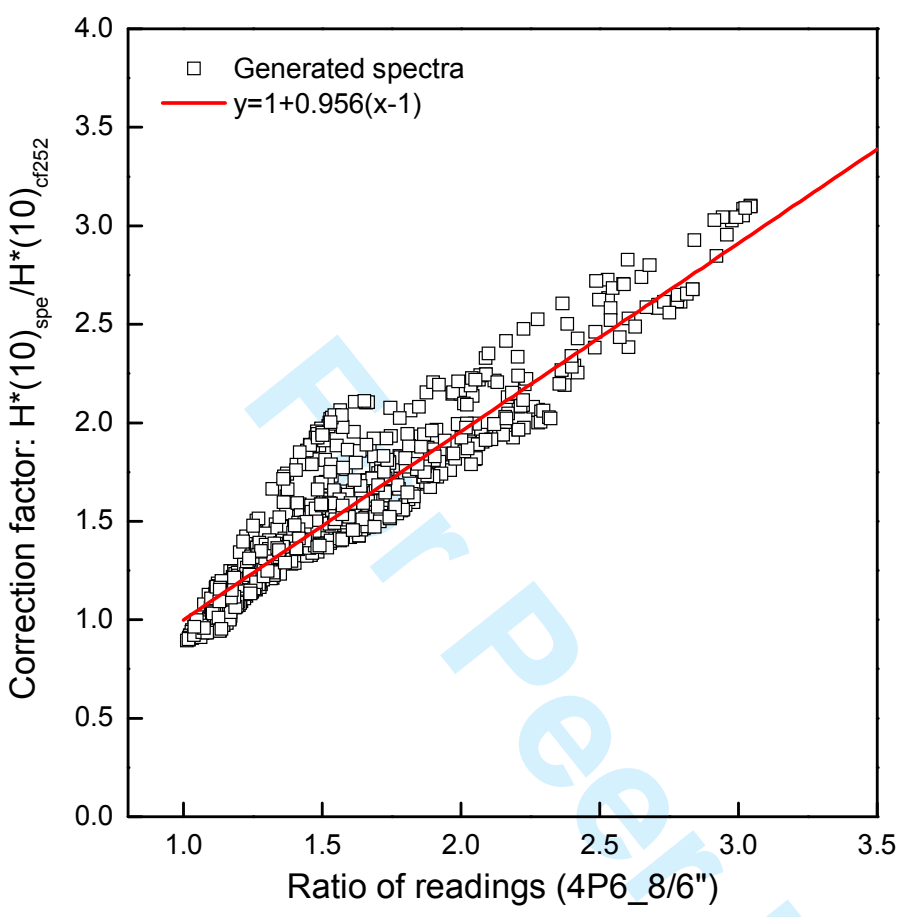

